Draft for PRE

\title{
Hurst Exponents For Short Time Series
}

\author{
Jingchao Qi and Huijie Yang* \\ Business School, University of Shanghai for Science and Technology, \\ Shanghai 200093, China
}

(Dated: November 14, 2012)

\begin{abstract}
A new concept, called balanced estimator of diffusion entropy, is proposed to detect scalings in short time series. The effectiveness of the method is verified by means of a large number of artificial fractional Brownian motions. It is used also to detect scaling properties and structural breaks in stock price series of Shanghai Stock market.
\end{abstract}

PACS numbers: 05.45.-a, 05.40.-a, 89.75.-k

Keywords: short time series; scaling; diffusion entropy

\section{INTRODUCTION}

Scale invariance has been found to empirically hold for a number of complex systems [1]. Consider a stochastic trajectory $X(t)$, whose statistical properties are described by the probability distribution function (PDF) of the displacements, $p(x, t)$. The stochastic process represented by $X(t)$ behaves scale-invariant, provides the PDF satisfies,

$$
p(x, t)=\frac{1}{t^{\delta}} F\left(\frac{x}{t^{\delta}}\right),
$$

where $\delta$ is the scaling exponent. Ordinary statistical mechanics is intimately related to the Central Limit Theorem [2], which implies the Gaussian form of the function $F(\cdot)$ and $\delta=0.5[3]$. By using the the scaling exponents one can describe quantitatively the deviations from ordinary mechanics, and consequently assess the real physical nature of a phenomenon. But evaluation of the scaling exponents meets several challenges.

How to evaluate a reliable scaling is not a trivial task. Variance-based methods are used widely in literature to calculate the scaling exponents [4], in which there exists an assumption of the time dependence of the variance $\operatorname{Var}(X(t))$ with the scaling exponent $\delta$, namely, $\operatorname{Var}(X(t)) \sim t^{2 \delta}$. For fractional Brownian motions it is valid, but there are scaling processes such as Lévy flights for which the second moment diverges or Lévy walks for which the second moment satisfies a scaling relation $\operatorname{Var}(X(t)) \sim t^{2 H}$ with $H \neq \delta$, i.e, the relationships are violated [5]. Several efforts have been done to develop complementary methods to evaluate reliable scaling exponents [6, 7]. To cite an example, from the PDF one can calculate the Shannon entropy, $S(t)=-\int p(x, t) \ln p(x, t) d x$, which is originally identified as diffusion entropy by Scafetta et al. [6]. It is proved that the diffusion entropy can provide simultaneously reliable values of $\delta$ for fractional Brownian motions and Levy processes.

*Electronic address: hjyang@ustc.edu.cn Corresponding author
For a real-world stochastic process, the PDF is generally not known. One can count how often the value $x$ appears in the data set of trajectory $X(t)$. Denoting the number with $n(x)$, the PDF can be estimated with the relative frequency, $\frac{n(x)}{N} . N$ is the total size of the data set. In many situations only small data sets from which to infer PDF are available. What is more, for a stochastic process with a large amount of data sets, there exist generally structural breaks in the trajectory due to emergent strikes from environments and/or the system's transition to a contrasting dynamical regime. To cite examples, a stock market is shocked frequently by currency and tax policies, before and after an earthquake the earth motion may stay in different dynamical regimes. We should separate the data sets into many sub-sets to detect the behaviors at different structural patterns. A small value of $N$ may induce large statistical fluctuations or even bias to physical quantities as the PDF, entropy, moments and so on. In a recent novel paper, Bonachela et al. recall the search for improved estimators of entropy for small data sets [8]. They propose also a new "balanced estimator" that out-performs other currently available ones when the data sets are small and $p(x, t)$ are not close to zero.

Stimulated by the two mentioned efforts, in the present paper a new concept is introduced, called Balanced Estimator of Diffusion Entropy (BEDE), in which the balanced estimator of entropy is used to replace the original form in the diffusion entropy. This concept is used to find scalings and structural breaks in artificial and empirical series. Firstly, we review briefly the concepts of diffusion entropy and balanced estimator, and introduce consequently the concept of BEDE. Secondly, the effectiveness of the BEDE in detecting scalings in short time series are verified by means of a large amount of fractional Brownian motions. Finally, we detect the scalings and structural breaks in the stock price series of Shanghai Stock Market. 


\section{METHODS AND MATERIALS}

To keep the description self-contained, we review briefly the concepts of diffusion entropy and balanced estimator.

\section{A. Diffusion Entropy}

Let us consider a one-dimensional stationary time series,

$$
\xi_{1}, \xi_{2}, \cdots, \xi_{N}
$$

$N$ is the length of the series. All the possible segments with length $s$ read,

$$
X_{i}=\left\{\xi_{i}, \xi_{i+1}, \cdots, \xi_{i+s-1}\right\}, i=1,2, \cdots, N-s+1 .
$$

Now we regard the length $s$ as time, the vector $X_{i}$ can be regarded as a stochastic trajectory of a particle starting from its initial position $X_{i}(0)=0$. By this way, the time series (2) is mapped to an ensemble containing $N-s+1$ realizations of a stochastic process. The displacements read,

$$
x_{i}(s)=\sum_{j=1}^{s} X_{i}(j)=\sum_{j=i}^{i+s-1} \xi_{j}, i=1,2, \cdots, N-s+1 .
$$

One can divide the displacement interval where the particle appears into $M(s)$ bins, and reckon the number of the particle's occurrences in each bin at time $s$. We denote the numbers with $N_{j}(s), j=1,2, \cdots, M(s)$. The PDF can be naïvely approximated by the relative frequency,

$$
p(j, s) \sim \hat{p}(j, s)=\frac{N_{j}(s)}{N-s+1}, j=1,2, \cdots, M(s) .
$$

The entropy of the diffusion process is consequently determined, which reads,

$$
S_{D E}(s) \sim S_{D E}^{\text {naive }}(s)=-\sum_{j=1}^{M(s)} \hat{p}(j, s) \ln [\hat{p}(j, s)] .
$$

This entropy is based upon the diffusion process constructed from the original series (2), for this reason is called Diffusion Entropy (DE) [9].

The key step in calculation of the DE is how to choose the size of the bins, $\epsilon(s)$. The easiest way is to assume it to be a fraction of the square root of the variance of the original series (2) and independent of $s$.

Now we assume the time series behaves scaleinvariance, namely, $p(j, s)$ obeys the relation (1),

$$
\begin{aligned}
& p(j, s)=\frac{1}{s^{\delta}} F\left(\frac{x_{\min }(s)+(j-0.5) \epsilon(s)}{s^{\delta}}\right), \\
& j=1,2, \cdots, M(s),
\end{aligned}
$$

where $x_{\min }(s)$ is the smallest value of displacement, i.e., $x_{\min }(s)=\min \left[x_{1}(s), x_{2}(s), \cdots, x_{N}(s)\right]$. Let us plug Eq.(7) into Eq.(6). A simple computation leads to,

$$
S_{D E}(s)=A+\delta \ln (s),
$$

where $A=\int_{-\infty}^{+\infty} d y F(y) \ln [F(y)]$.

The simple relation of Eq.(8) can be used to detect scalings in time series. It is the first tool yielding the correct scaling in both the Gauss and the Lévy statistics. For this reason, it attracts special attentions from diverse research fields [10].

\section{B. Balanced Estimator For Diffusion Entropy}

From the relation Eq.(5) we have the ensemble average,

$$
\langle\hat{p}(j, s)\rangle=\frac{\left\langle N_{j}(s)\right\rangle}{N-s+1}=p(j, s) .
$$

In other words, the frequencies $\hat{p}(j, s)$ approximate the probabilities with certain statistical error (variance) but without any systematic error (bias). The frequencies $\hat{p}(j, s)$ are unbiased estimators of the probabilities $p(j, s)$.

However, there is an important difference between $S_{D E}(s)$ and $S_{D E}^{\text {naive }}(s)$ in $E q .(6)$ stemming from the nonlinear nature of the entropy functional. Defining an error variable, $\mu(j, s)=\frac{\hat{p}(j, s)-p(j, s)}{p(j, s)}$, and replacing $p(j, s)$ in $S_{D E}$ by its value in terms of $\mu(j, s)$ and $\hat{p}(j, s)$, a straightforward algebraic leads [11],

$$
S_{D E}(s)=S_{D E}^{\text {naive }}+\frac{M(s)-1}{2(N-s+1)}+\mathcal{O}(M(s)) .
$$

The leading order of error, $\frac{M(s)-1}{2(N-s+1)}$, is a significant error for small $N-s+1$ and vanishes only as $(N-s) \rightarrow \infty$. Consequently, $S_{D E}^{\text {naive }}(s)$ is a biased estimator of $S_{D E}(s)$, i.e., it deviates from the true entropy not only statistically but also systematically.

An improved estimator of $S_{D E}(s)$ should reduce the bias or the variance as possible, which can be formulated as follows. Defining $S_{D E}[p(j, s)] \equiv-p(j, s) \ln [p(j, s)]$, we have $S_{D E}(s)=\sum_{j=1}^{M(s)} S_{D E}[p(j, s)]$. We want to find an estimator,

$$
\hat{S}_{D E}(s) \equiv \sum_{j=1}^{M(s)} \hat{S}_{D E}[n(j, s)]
$$

so that the bias,

$$
\Delta_{\text {bias }}^{2}(s)=\left(\left\langle\hat{S}_{D E}(s)\right\rangle-S_{D E}(s)\right)^{2}
$$

or the mean squared deviation

$$
\Delta_{\text {stat }}^{2}(s)=\left\langle\left(\hat{S}_{D E}(s)-\left\langle\hat{S}_{D E}(s)\right\rangle\right)^{2}\right\rangle
$$


or a combination of both are as small as possible.

Ignoring correlations between the elements of the distribution, $n(j, s), j=1,2, \cdots, M(s)$, the problem can be reduced to minimize simultaneously the bias and the variance for each summand,

$$
\Delta_{\text {bias }}^{2}[p(j, s)]=\left(\left\langle\hat{S}_{D E}[n(j, s)]\right\rangle-S_{D E}[p(j, s)]\right)^{2}
$$

and

$$
\Delta_{\text {stat }}^{2}[p(j, s)]=\left\langle\left(\hat{S}_{D E}[n(j, s)]-\left\langle\hat{S}_{D E}[n(j, s)]\right\rangle\right)^{2}\right\rangle,
$$

where the probability $p(j, s) \in[0,1]$, and $n(j, s) \in$ $\{0,1,2, \cdots, N-s+1\}$ is binomially distributed. To balance the errors, we minimize the average error over the whole range of $p(j, s) \in[0,1]$,

$\Delta^{2}(j, s)=\int_{0}^{1} d p(j, s) \cdot w[p(j, s)] \cdot\left[\Delta_{\text {bias }}^{2}(j, s)+\Delta_{\text {stat }}^{2}(j, s)\right]$,

where $w[p(j, s)]$ is a suitable weight function that specific problem depends. Without extra knowledge of the probability values, one can consider a simple case of $w[p(j, s)]=1$. Inserting Eq.(14) and Eq.(15) into $E q .(16)$, the average error is given by,

$$
\begin{aligned}
& \Delta^{2}(j, s)= \\
& \int_{0}^{1} d p(j, s)\left\{\sum_{n(j, s)=0}^{N-s+1} P_{n(j, s)}[p(j, s)] \hat{S}_{D E}^{2}[n(j, s)]\right. \\
& +S_{D E}^{2}[p(j, s)] \\
& \left.-2 S_{D E}[p(j, s)]\left(\sum_{n(j, s)=0}^{N-s+1} P_{n(j, s)}[p(j, s)] \hat{S}_{D E}[n(j, s)]\right)\right\},
\end{aligned}
$$

where $P_{n(j, s)}[p(j, s)]$ is the binomial distribution,

$$
\begin{aligned}
& P_{n(j, s)}[p(j, s)]=\frac{[N-s+1] !}{n(j, s) ![N-s+1-n(j, s)] !} \times \\
& \quad[p(j, s)]^{n(j, s)} \cdot[1-p(j, s)]^{N-s+1-n(j, s)} .
\end{aligned}
$$

The goal is to determine all the numbers $\hat{S}_{D E}[n(j, s)], j=1,2, \cdots, N-s+1$ which leads to the minima of the average error. The necessary condition is that all the partial derivatives vanish, i.e.,

$$
\frac{\partial \Delta^{2}(j, s)}{\partial \hat{S}_{D E}[n(j, s)]}=0, n(j, s)=1,2, \cdots, N-s+1 .
$$

Detailed computation leads to,

$$
\begin{aligned}
& \hat{S}_{D E}[n(j, s)] \\
& =(N-s+2) \int_{0}^{1} d p(j, s) P_{n(j, s)}[p(j, s)] \cdot S_{D E}[p(j, s)] \\
& =\frac{n(j, s)+1}{N-s+3} \sum_{k=n(j, s)+2}^{N-s+3} \frac{1}{k} .
\end{aligned}
$$

The final improved estimator of diffusion entropy reads,

$$
\hat{S}_{D E}(s)=\frac{1}{N-s+3} \sum_{j=1}^{M(s)}\left[N_{j}(s)+1\right] \cdot \sum_{k=N_{j}(s)+2}^{N-s+3} \frac{1}{k},
$$

called Balanced Estimator of Diffusion Entropy (BEDE) in this paper.

\section{Materials}

\section{Fractional Brownian Motions}

Fractional Brownian motions (fBm) [12] are used to verify the effectiveness of BEDE in detecting scalings in short time series. A fBm is a continuous-time Gaussian process depending on the Hurst parameter $0 \leq H \leq 1$. The $\mathrm{fBm}$ is self-similar in distribution and the variance of the increments is given by $\operatorname{Var}(f B m(t)-f B m(s)) \sim$ $|t-s|^{2 H}$. The program wfbm.m in Matlab ${ }^{\circledR}$ is used to generate the $\mathrm{fBm}$ series.

\section{Shanghai Stock Exchange Indices}

The empirical data are the time series of stock price indices from the Shanghai Stock Exchange (SSE) 13], the world's 5th largest stock market by market capitalization at US2.7 trillion as of Dec 2010. The current exchange was established on November 26, 1990 and was in operation on December 19 of the same year. We collect totally 134 closed stock price series starting from the end of the year 1995 to the end of June, 2010, in which the numbers of the stocks distribute in the categories of industry, business, real estate, public utility, and comprehension are 64,27,12, 12 and 19, respectively. We consider also the stock price indices of the five categories from December 6,1994 to June 30, 2010. The SSE index series starts from December 19,1990 and ends at June 30, 2010.

For a closed stock price series, $p(t)$, one can construct the corresponding return series,

$$
r(t)=\frac{\log _{a}[p(t+\Delta t)]}{\log _{a}[p(t)]} .
$$

In the calculations, $\Delta t$ is selected to be 5 , i.e., weekly return ratio is considered.

\section{RESULTS}

Fig.1 presents several typical examples of comparison between BEDE and DE. For the three generated $\mathrm{fBm}$ series, (a)-(c), with $H=0.7$ and lengths 650,650, 4000, respectively, in a wide range of $s$, the BEDEs obey almost perfect linear relations versus $\ln (s)$ as shown in (d)-(f), i.e., the scalings are all perfectly rendered out. The estimated values of the scalings ( the slope of the BEDE curve) are $0.70,0.67$ and 0.71 , which can be regarded as the same with the expected value of $H=0.7$. However, for the short series in (a) and (b), with the increase of time, the DE curves tend to bent down and the deviations from the linear relations of $B E D E$ versus $\ln (s)$ 

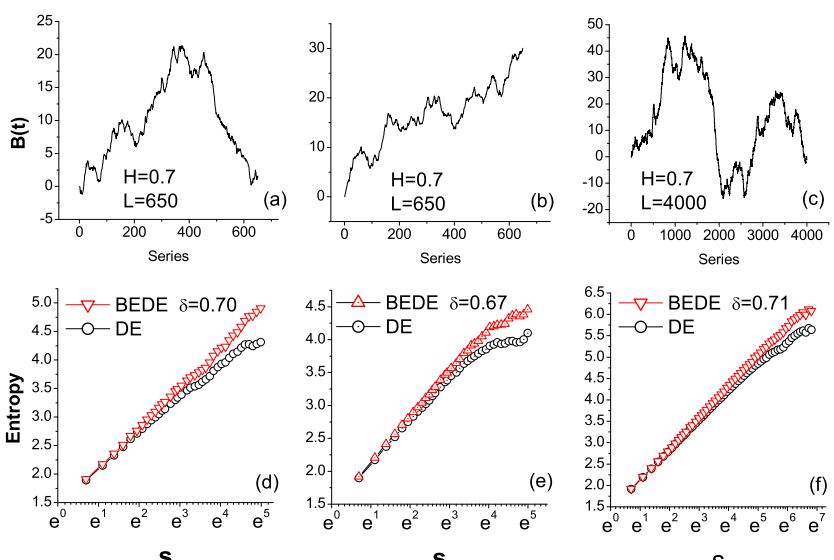

$\mathrm{s}$
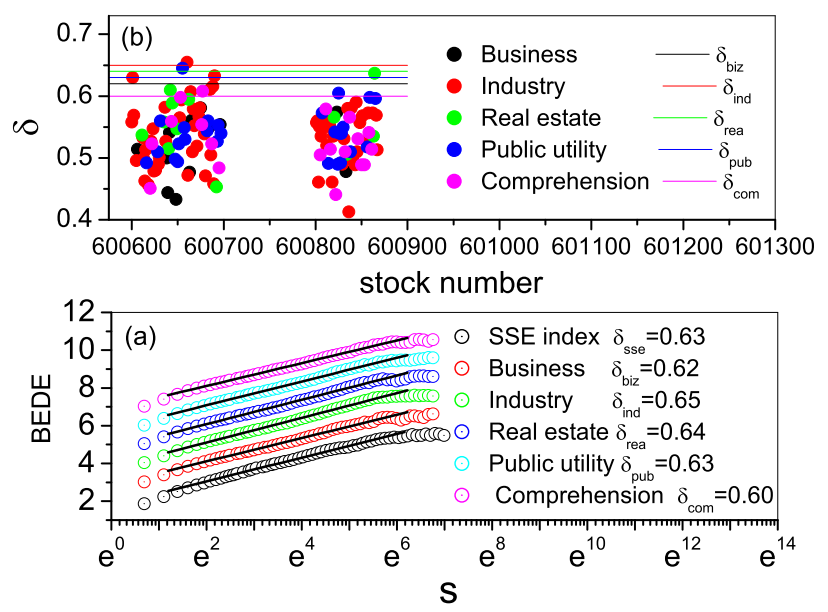

FIG. 1: (Color online) Typical examples of comparison between BEDE and DE. (a)-(c) Generated fBm motions with $H=0.7$ and lengths 650,650,4000, respectively. (d)-(f) In a wide range of $s$, the BEDEs obey almost perfect linear relations versus $\ln (s)$. The estimated values of the scalings ( the slope of the BEDE curve) are $0.70,0.67$ and 0.71 . With the increase of time, the DE curves tend to bent down and the deviations from the linear relations of $B E D E$ versus $\ln (s)$ become more and more significant. With the increase of length, e.g., 4000, the DE curve is corrected significantly to be much more closer to the BEDE curve in a wider range of $\ln (s)$, as shown in $(f)$.
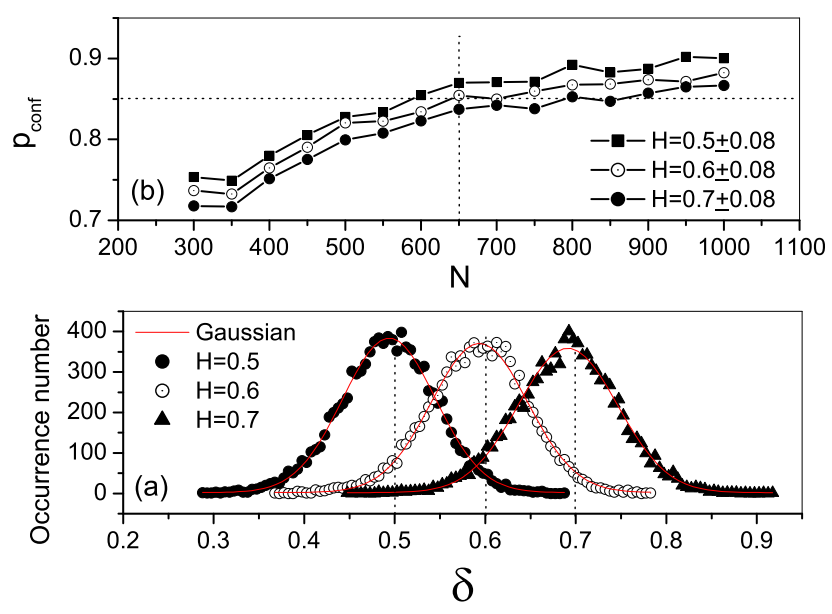

FIG. 2: (Color online) Confidence of BEDE-based scaling estimation. (a) $10^{4}$ series with $N=650$ and $H=$ $0.5,0.6$ and 0.7 are generated, respectively. The scaling estimations distribute normally and center at the expected values of $\delta=0.5,0.6$ and 0.7 . (b) The relation of certainty level versus series length at a specified confidence interval $\Delta H=0.08$.
FIG. 3: (Color online) BEDE-based scaling estimations for the SSE index, and the indices of the five catalogues including industry, business, real estate, public utility, and comprehension. (a) In a wide range of $s$, the relations of BEDE versus $\ln (s)$ obey a linear-law. The scaling exponents are $\delta_{\text {sse }}=0.63, \delta_{\text {biz }}=0.62, \delta_{\text {ind }}=0.65, \delta_{\text {rea }}=$ $0.64, \delta_{\text {pub }}=0.63$, and $\delta_{\text {com }}=0.60$, respectively . (b) The scaling estimations for the selected 134 stocks. The scaling exponent for each catalogue is significantly larger compared with that of the stocks included in the corresponding catalogue.

become more and more significant. With the increase of length, e.g., 4000 in Fig.1(c), the DE curve is corrected significantly to be much more closer to the BEDE curve in a wider range of $\ln (s)$, but at the region with larger values of $s$ it still bents down with an unacceptable bias. Hence, for a series with short length as $N \sim 650$, the calculated values of $D E$ have unacceptable errors due to bias. The curve of $D E$ versus $\ln (s)$ can not detect correctly the scaling at all, while the BEDE can give perfect estimations of entropy even for considerable large of $s$, namely, a small set of data $(N-s+1$ records). We must correct the bias in entropy by means of the BEDE in analyzing the stock price series of SSE, which has only a short history of about 15 years ( $\sim 10^{3}$ in length).

For a specific value of $H$, one can generate a large amount of $\mathrm{fBm}$ series. It is found that the scaling estimations distribute normally, as shown in Fig.2(a) a typical example, in which totaly $10^{4}$ series with $N=650$ and $H=0.5,0.6$ and 0.7 are used. By specifying a confidence interval $[H-\Delta H, H+\Delta H]$ the corresponding level of certainty $p_{\text {conf }}$ is determined so that $p_{\text {conf }} \cdot N_{\text {conf }}$ estimations occur in the confidence interval. $N_{\text {conf }}$ is the total number of the generated $\mathrm{fBm}$ series. Fig.2(b) shows the relation of the certainty level $p_{\text {conf }}$ versus the series length $N$. At the beginning, with the increase of $N, p_{\text {conf }}$ increases rapidly, while when $N$ becomes large $p_{\text {conf }}$ tends to saturate to a high value. Accordingly, we 


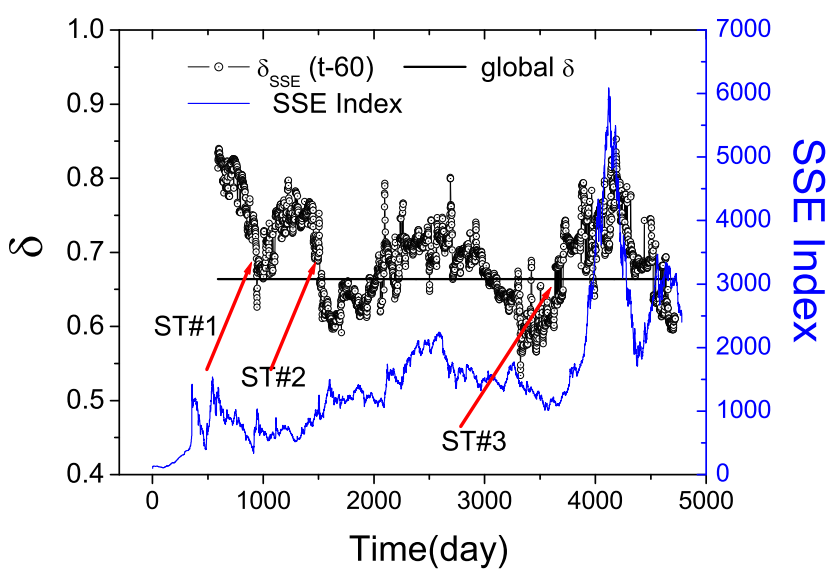

FIG. 4: (Color online) Evolution of local scaling estimation. Segment length is chosen to be 650 . The SSE index series and the $\delta$ evolution curve are matched with $\Delta t=60$. The value of $\delta$ distributes in a wide interval from 0.53 to 0.85 . The scaling for the total series is 0.63 . There exist globally four peaks covering 355, 676, 1704 and 1396 data points, namely, persisting roughly $18,34,85$ and 70 months, respectively. The significant transitions marked with ST\#1, ST\#2 and ST\#3 correspond to the bull market in the duration from July 29, 1994 to Sept. 13,1994, the bull market in the duration from Jan.19,1996 to May 12,1997, and the RSSLCinduced increase of SSE index for about 24 months (staring from May 9,2005), respectively.

select $N=650$ in the following calculations in detecting local scaling behaviors of the stock series in SSE stock market.

We calculate the BEDEs for the Shanghai Stock Exchange index (SSE), and the indices of the five catalogues including industry, business, real estate, public utility, and comprehension. In a wide range of $s$, the relations of BEDE versus $\ln (s)$ obey a linear-law. Hence, there exist almost perfect self-similarities and the scaling exponents are $\delta_{\text {sse }}=0.63, \delta_{\text {biz }}=0.62, \delta_{\text {ind }}=0.65, \delta_{\text {rea }}=$ $0.64, \delta_{\text {pub }}=0.63$, and $\delta_{\text {com }}=0.60$, respectively (see Fig.3(a)). The scaling estimations for the selected 134 stocks are also calculated, as shown in Fig.3(b). The scaling exponent for each catalogue is significantly larger compared with that of the stocks included in the corresponding catalogue. Though each specific stock is almost not predictable, the catalogue it belongs to, i.e., a combination of the stocks in the catalogue is much more predictable.

For the SSE series, $\left\{r_{S S E}(1), r_{S S E}(2), \cdots, r_{S S E}(N)\right\}$, one can calculate the scaling exponents for all the segments of $\left\{r_{S S E}(t-s+1), r_{S S E}(t-s+2), \cdots, r_{S S E}(t)\right\}, t=$ $s, s+1, \cdots, N$, denoted with $\delta_{S S E}(t-\Delta t)$, which are employed in the present work to represent the local scalings of the SSE series, as shown in Fig.4. The value of $s$ is chosen to be 650 . Assuming a structural break occurs at time $t$, only when the segment covers a certain number of data after the time $t$, the contribution from the break's occurrence becomes significant and detectable. We introduce the parameter $\Delta t$ to describe this kind of delay effect.

It is found that in the more than ten years duration the value of $\delta$ distributes in a wide interval from 0.53 to 0.85 . The scaling for the total series is 0.63 , a value comparatively closer to the lower bound 0.53 . What is more, though there exist rich fine-structures with locally abrupt changes, there are globally four peaks covering $355,676,1704$ and 1396 data points, namely, persisting roughly 18,34, 84 and 69 months, respectively.

It is reasonable to believe that important events, such as policies and/or emergencies, may lead speedy transitions of a stock market from lower(higher) to higher(lower) predictable. From the evolutionary curve of $\delta$, one can find three sharp transitions marked with red arrows and denoted with $S T \# 1 S T \# 2$ and $S T \# 3$, respectively. The distances between the successive transitions are about 17.5 and 112.5 months. By comparing with the important events occurring in the history of the SSE market [14], the value of $\Delta t$ is determined to be 60 . By this way the stock series and the $\delta$ evolutionary series are matched along time, as shown in Fig.4.

The first transition, $S T \# 1$, corresponds to the bull market in the duration from July 29, 1994 to Sept. 13,1994. Before this bull market, the market has suffered from a 17-month-duration of decrease. The China Securities Regulatory Commission (CSRC) issues three special policy regulation items to bailout the stock market. Accordingly, the SSE index increases rapidly from 325 to 1052 within one and half month (reaches the record at Sept. 13,1994).

The second transition, $S T \# 2$, matches with the bull market in the duration from Jan.19,1996 to May 12,1997, in which the stock index rises up to 1464 from 512. At the time speculating blue chip stocks tends to dominate the investment concept. The Shenzhen Development Bank and Sichuan Changhong become successively the leading stocks in the Shenzhen Stock Exchange market and The Shanghai Stock Exchange market, respectively. Stimulated by the two stocks, the SSE market becomes high active and after the National day of China, the prices for almost all stocks increase rapidly. The CSRC issues successively some policy regulation items to cool down the stock market and expounds in-detail the irrational state of the stock market.

Starting from June 13,2001, the day a local maxima occurs at level 2242, a decreasing process persists about 48 months, during which a bouncing maxima occurs at level 1778 at April 6, 2004. At May 9,2005 the reform of the shareholder structure of listed companies (RSSLC) is conducted, which induces a persistent increase of SSE index for about 24 months. This event accords with the third transition, $S T \# 3$. Then the persistent increase is disturbed to a chaotic state by the escalation of stamp 
tax at May 30,2007.

\section{CONCLUSIONS}

Scaling invariance holds in a large amount of complex systems, but the evaluation of scaling is still a challenge task. Theoretically, variance-based methods can not detect correctly the scalings for Levy processes. Empirically, time series are usually not long enough to derive a reliable scaling exponent. What is more, in a long time series there exist usually structural breaks. In literature, diffusion entropy is developed to detect reliable scaling exponents for long time series. In the present paper, the balanced estimation of entropy for short time series is introduced to the diffusion entropy to find reliable scalings embedded in short time series.

This method can give reliable scalings even for short time series with length $\sim 10^{2}$. It is used to detect the scalings embedded in totally 134 stocks in SSE market.
The scaling exponent for each catalogue is significantly larger compared with that for the specific stocks included in this catalogue.

We detect also the local scalings in the SSE index series. The scalings varies in a large interval from 0.53 to 0.85 . Three speedy transitions from high(low) to low(high) values of $\delta$ occur in the evolutionary curve of $\delta$, which are used to match the $\delta$ curve with the closed price curve along time.

\section{Acknowledgements}

The work is supported by the National Science Foundation of China under Grant Nos. 10975099 and 10635040, the Program for Professor of Special Appointment (Eastern Scholar) at Shanghai Institutions of Higher Learning, and the Shanghai leading discipline project under grant No.S30501.We thank the reviewers for their stimulating and constructive comments and suggestions.
[1] H.E. Stanley, L.A.N. Amaral, P. Gopikrishnan, P. Ch. Ivanov, T.H. Keitt, V. Plerou, Physica A 281,60(2000).

[2] A.I. Khinchin, Mathematical Foundations of Statistical Mechanics (Dover Publications, Inc. New York, 1949).

[3] N. Goldenfeld, Lectures on Phase Transitions and the Renormalization Group ( Perseus Book, Reading, Massachusetts,1985).

[4] B.B. Mandelbrot, Fractals; form, chance and dimension, W.H. Freeman and Co., San Francisco(1977); C. K. Peng, S.V. Buldyrev, S. Havlin, M. Simons, H. E. Stanley and A. L. Golberger, Phys. Rev. E, 49, 1685 (1994).

[5] M.F. Shlesinger, J. Klafter and B.J. West, Phys. Rev. Lett. 58, 1100-03 (1987).

[6] N. Scafetta, P. Hamilton and P. Grigolini , Fractals 9, 193 (2001); P. Grigolini, L. Palatella and G. Raffaelli, Fractals 9, 439 (2001).

[7] M. Ignaccolo, P. Grigolini, B. J. West, E-print arXiv:1004.0372v1 [physics.data-an] 2 April 2010.

[8] J. A. Bonachela, H. Hinrichsen, and M. A. Muñoz, J.
Phys. A: Math. Theor. 41,202001(2008).

[9] N. Scafetta, and P. Grigolini, Phys. Rev. E 66, 036130(2002).

[10] N. Scafetta, Fractal and Diffusion Entropy Analysis of Time Series: Theory, concepts, applications and computer codes for studying fractal noises and Lévy walk signals, VDM Verlag Dr. Müller (May, 2010).

[11] M. S. Roulston, Physica D 125, 285 (1999).

[12] P. Abry, F. Sellan, Appl. and Comp. Harmonic Anal.,3(4),377(1996); J.-M Bardet,G. Lang, G. Oppenheim, A. Philippe, S. Stoev, and M. S. Taqqu, "Generators of long-range dependence processes: a survey", Theory and applications of long-range dependence, Birkhäuser, pp. 579-623.

[13] http://en.wikipedia.org/wiki/Shanghai_Stock_Exchange

[14] J. Liu, P. Zhang, K. Zhou, and J. Zhang, Beijing Modern Business Today, May 19,2006. 